\title{
Implantes con grabado ácido y pasivado químico: estudio preliminar sobre carga precoz
}

\author{
MARTINEZ-GONZALEZ JM JM $^{\mathrm{a}}$ * \\ BARONA DORADO C ** \\ FLOREZ RODRIGUEZ $\mathbf{M}^{\mathrm{a}} * * *$ \\ DONADO AZCARATE A **** \\ SUAREZ QUINTANILLA JM ${ }^{\mathrm{a}} * * * * *$
}

\begin{abstract}
Martínez-González JM ${ }^{\mathrm{a}}$, Barona Dorado C, Flórez Rodríguez $\mathrm{M}^{\mathrm{a}}$, Donado Azcárate A, Suárez Quintanilla JMª . Implantes con grabado ácido y pasivado químico: estudio preliminar sobre carga precoz. Av Periodon Implantol. 2001; 13, 3: 9-15.
\end{abstract}

\begin{abstract}
RESUMEN
Presentamos un estudio sobre un nuevo sistema de implantes en los que su superficie se encuentra tratada mediante un procedimiento de grabado ácido y pasivado químico. El número de pacientes que integraron este ensayo fue de quince, a los cuales se les colocaron un total de setenta implantes sobre situaciones diferentes, en cuanto a calidad y cantidad óseas se refiere. Los resultados ofrecen, en una primera fase, un $95,71 \%$ de éxito, permitiendo realizar la carga protésica en un tiempo aproximado de dos meses, lo que nos hace establecer como conclusiones preliminares que este tipo de superficie implantaria permite reducir el tiempo de espera de nuestros pacientes con unos resultados similares a los obtenidos con otras superficies más convencionales.
\end{abstract}

\section{PALABRAS CLAVE}

Implantes. Grabado ácido. Pasivado químico. Carga precoz.

\section{INTRODUCCIÓN}

La Implantología, al igual que otras áreas de la Odontoestomatología está sufriendo cambios constantes en su desarrollo debido a la aparición de nuevas tecnologias. Se puede observar, como los planteamientos iniciales propuestos por Branemark han venido modificándose hasta tal forma que algunos de los principios propuestos por este autor han quedado abandonados. Esta transformación puede atribuirse, en parte, a la aparición de nuevos diseños de implantes, tratamiento de sus superficies y un mejor conocimiento sobre la biología de la osteointegración.

Uno de los mayores retos a los que se enfrentan los implantólogos es el conseguir menores tiempos de reposo y posibilitar, de esta forma, que los pacientes puedan ser rehabilitados en tiempos más cortos. El tratamiento con implantes y su carga inmediata aparece ya en los estudios preconizados por Lederman ${ }^{1}$ en 1979 quien obtiene resultados satisfactorios. Otros autores han seguido en esta línea y han expuesto sus experiencias, con resultados similares, abriendo un nuevo camino en la disminución de los periodos de reposo ${ }^{2-4}$. Sin embargo, esta nueva forma de terapéutica implantológica encuentra limitaciones en sus indicaciones, estando dirigida fundamentalmente a rehabilitaciones en el sector anteroinferior.

Una situación intermedia se encuentra representada con los denominados, implantes de carga precoz, que también tratan de reducir el tiempo de reposo con-

* Profesor Titular de Cirugía. Facultad de Odontología. Universidad Complutense de Madrid.

** Profesora Colaboradora. Facultad de Odontología. Universidad Complutense de Madrid.

*** Servicio de Implantología Bucofacial. Hospital de Madrid.

**** Profesor Ayudante. Facultad de Odontología. Universidad Complutense de Madrid.

***** Profesor Titular de Cirugía. Facultad de Medicina y Odontología. Universidad de Santiago de Compostela. 
vencional a algo menos de la mitad, pero con la ventaja de ampliar sus indicaciones. En esta línea, el tratamiento de la superficie implantaria con grabado de distintos ácidos como $\mathrm{HCl}, \mathrm{H} 2 \mathrm{SO} 4, \mathrm{HNO} 3$, han favorecido esta aplicación.

El propósito de este trabajo es aportar los resultados iniciales del implante Defcon ${ }^{\circledR}$, que se caracteriza por su superficie implantaria tratada mediante grabado ácido y pasivado químico, lo que confiere la posibilidad de someter a carga precoz a estos implantes tras un período de reposo de dos meses.

\section{MATERIALES Y METODOLOGÍA}

El presente estudio ha sido realizado en el Servicio de Implantología del Hospital de Madrid sobre un total de quince pacientes a los que les fueron colocados 70 implantes de carga precoz Defcon .

El protocolo seguido consistió en la realización de historia y exploración clínica con montaje y estudio de modelos. Posteriormente se solicitaron los estudios radiológicos mediante técnicas panorámicas y tomográficas.

Una vez planificado el tratamiento, se solicitó el consentimiento a todos los pacientes para integrarse en el estudio. A cada uno de ellos, se les rellenó una ficha en la que se hizo constar: edad, sexo, localización de la zona a tratar, cantidad y calidad óseas, planificación protésica, longitud y diámetro de los implantes.

Éstos, son implantes cilíndricos roscados que se encuentran disponibles en tres diámetros de cuerpo y varias longitudes: serie 3 (diámetro 3,4 mm y longitu-

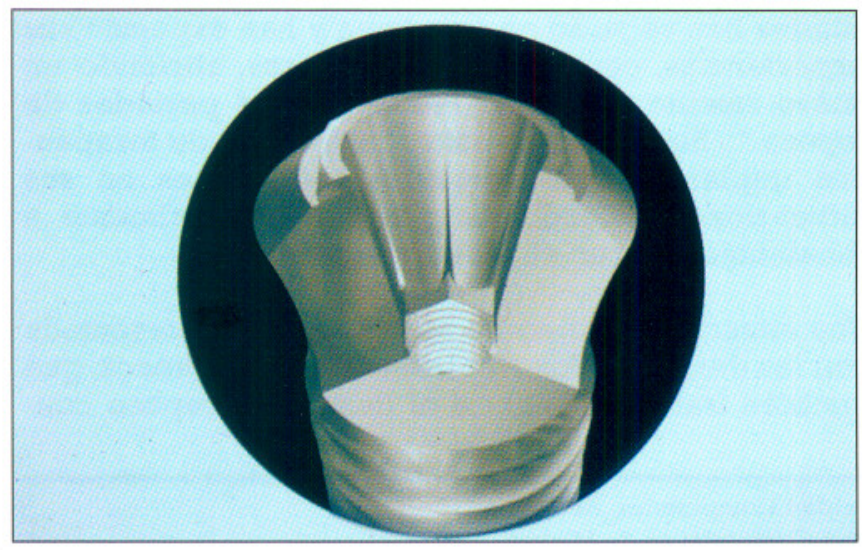

Fig. 1: Sistema multiconexión del implante Defcon $^{\otimes}$ caracterizado por conos externo e interno y hexágonos externo e interno.

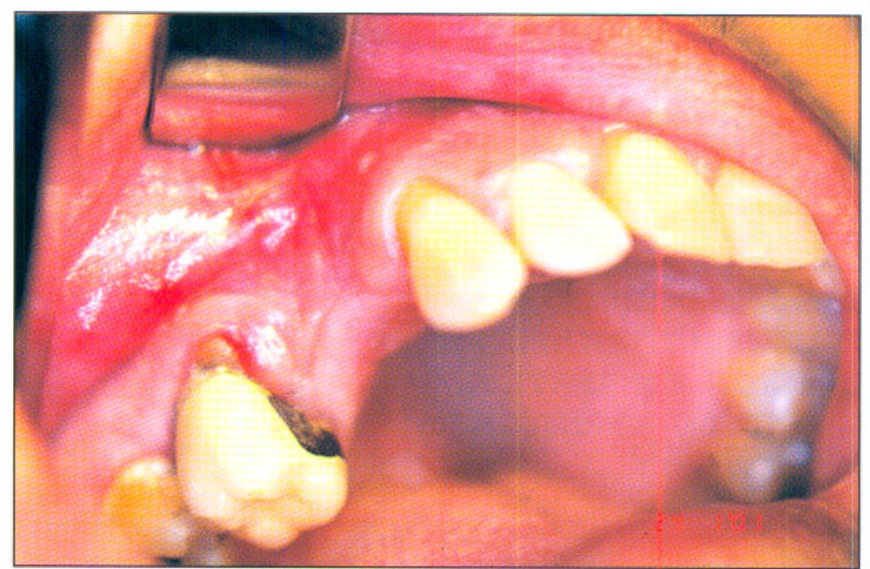

Fig. 2: Imagen clínica para colocación de dos implantes (serie 313) a nivel de premolares.

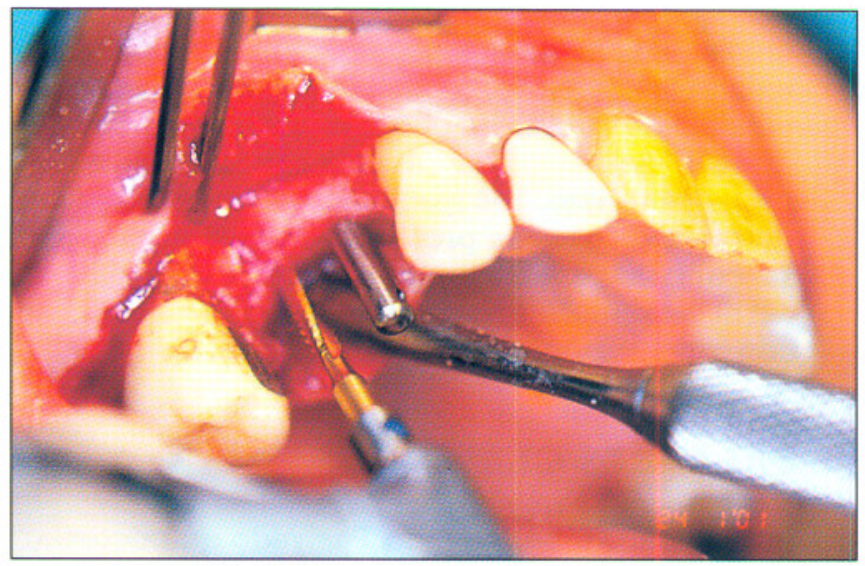

Fig. 3: Fase quirúrgica con paralelizador y utilización de la fresa piloto.

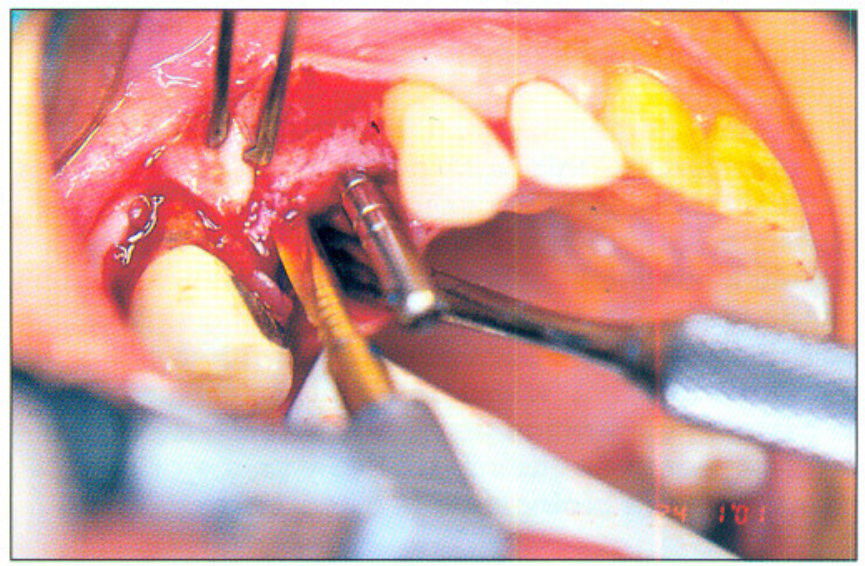

Fig. 4: Fase quirúrgica con utilización de la segunda fresa correspondiente a la serie 3.

des 10,13 y $16 \mathrm{~mm}$ ), serie 4 (diámetro $4 \mathrm{~mm}$ y longitudes 10,13 y $16 \mathrm{~mm}$ ) y serie 5 (diámetro $5,3 \mathrm{~mm}$ y longitudes de 8,10 y $13 \mathrm{~mm}$ ), teniendo además como particularidad sus sistema de multiconexión formada 


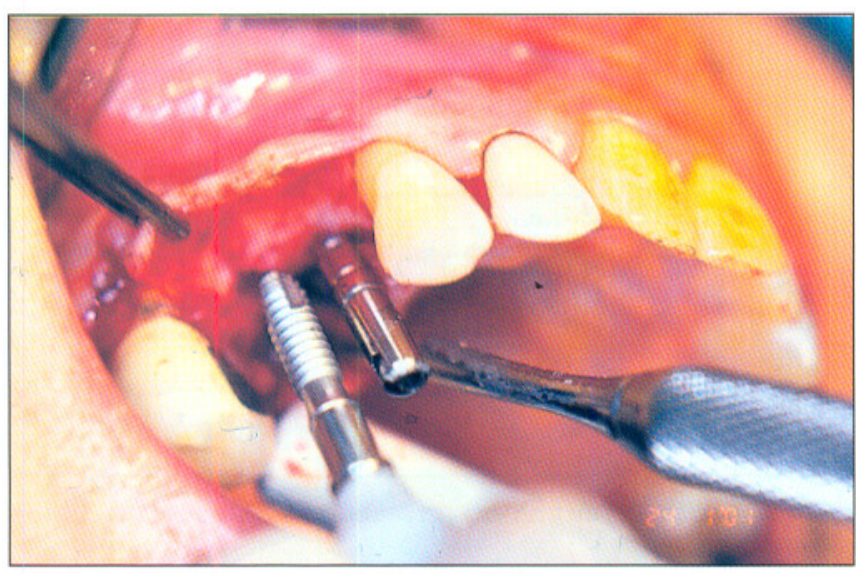

Fig. 5: Comienzo de la inserción del implante en el lecho óseo que puede realizarse de forma manual o rotatoria.

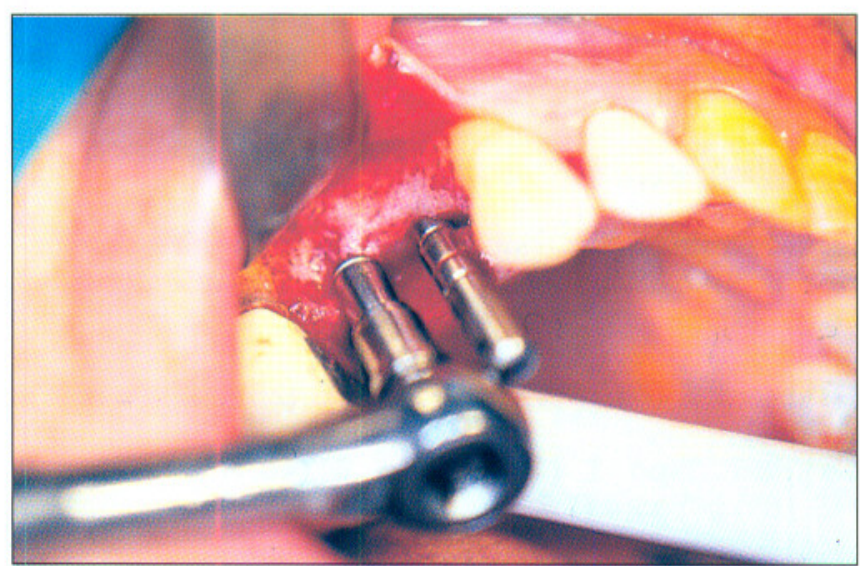

Fig. 6: Ultimas vueltas de roscado utilizando la carraca correspondiente.

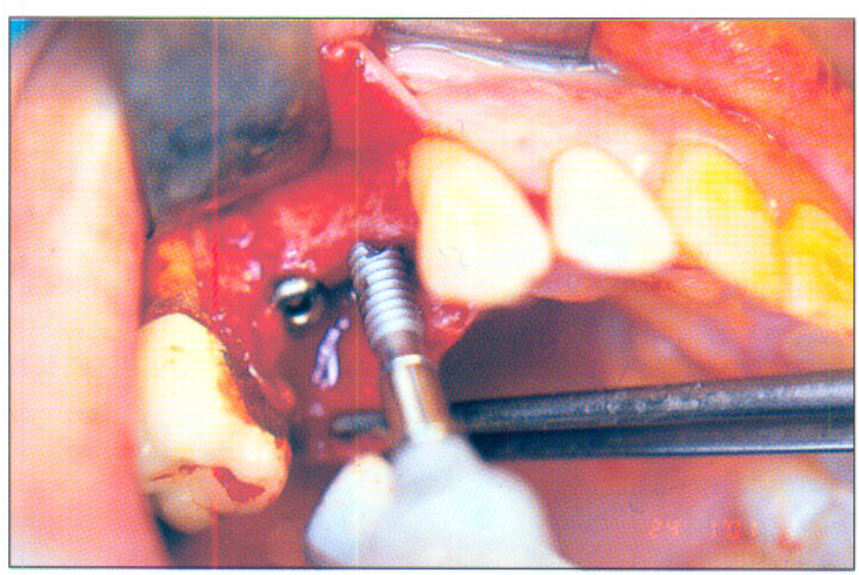

Fig. 7: Fase quirúrgica en la que se realiza la inserción del segundo implante.

por cuatro conexiones - cono externo e interno y hexágono externo e interno -. (Fig. $n^{\circ} 1$ )

El tratamiento quirúrgico consistió en el habitual para los tratamientos implantológicos de dos fases (sumer-

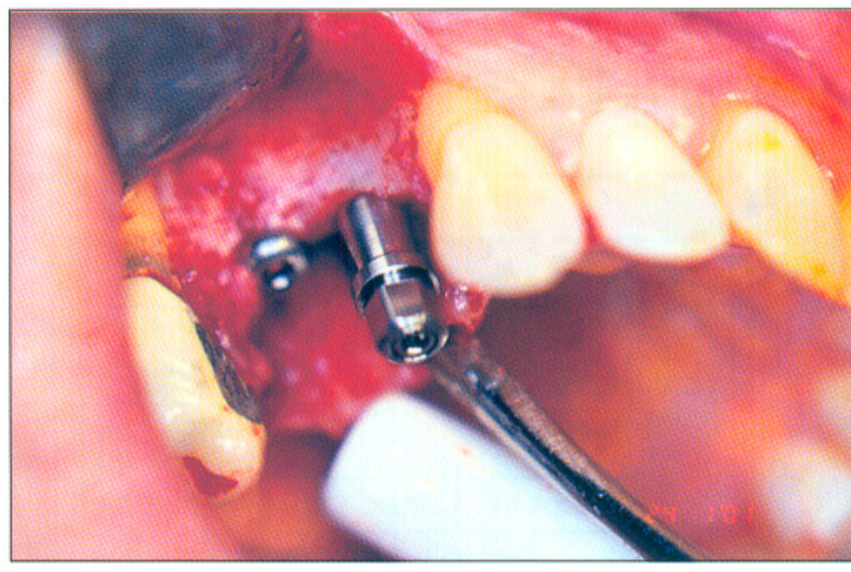

Fig. 8: Imagen clínica antes de proceder a la retirada del transportador del segundo implante.

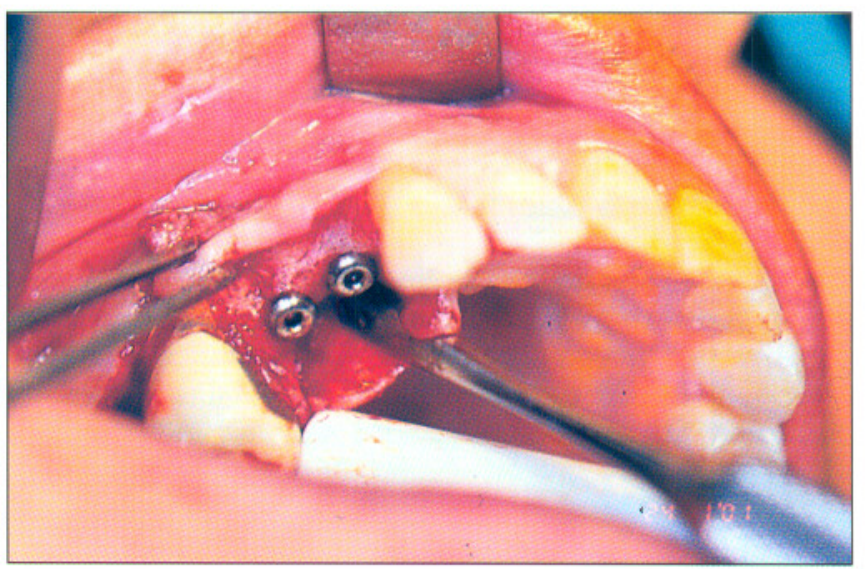

Fig. 9: Imagen clínica final una vez insertados los implantes.

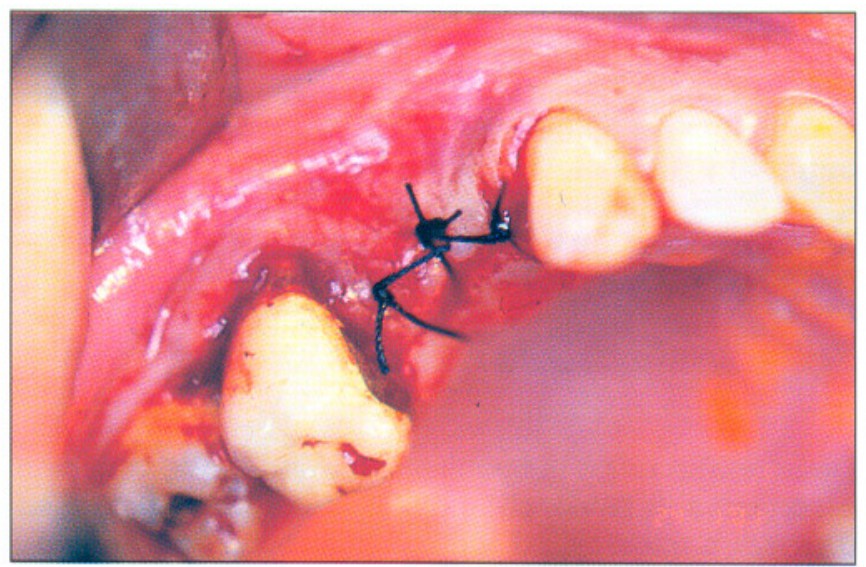

Fig. 10: Reposición del colgajo mucoperióstico y posterior sutura.

gido inicial y descubrimiento para colocación de cabezas de cicatrización).

Todos los pacientes fueron tratados bajo anestesia local combinada con sedación, sin presentarse ningún tipo de complicación durante el acto quirúrgico. 


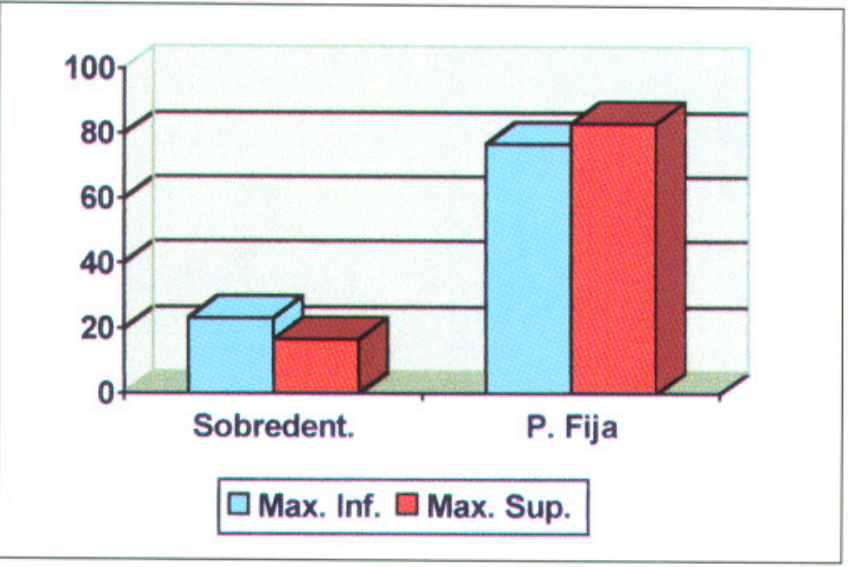

Fig. 11: Distribución de porcentajes según región anatómica y tratamiento protésico realizado.

Una vez preparado y desinfectado el campo, se procedió a la realización del colgajo mucoperióstico, colocación de férula quirúrgica para posicionamiento y diseño del lecho óseo con las fresas correspondiente al tratamiento planificado (Figs. $n^{\circ} 2-10$ )

Tras la inserción de los implantes se reposicionó el colgajo mucoperióstico, suturándose el mismo con seda de 000. Una vez finalizado, se anotó la terapéutica postoperatoria, aparición de complicaciones y hallazgos en los controles radiológicos realizados al mes y medio.

\section{RESULTADOS}

\section{Edad y sexo:}

La edad media de los pacientes que integraron este estudio fue de 59,06 años con un rango comprendido entre 40 y 81 años.

La distribución de acuerdo al sexo fue de trece mujeres $(86,66 \%)$, frente a dos varones $(13,33 \%)$.

\section{Región anatómica y tipo de rehabilitación (Figura $n^{\circ}$ 11):}

De la totalidad de los pacientes rehabilitados tan solo en un caso $(6,66 \%)$, se realizó tratamiento simultáneo de ambos maxilares. En el resto, hubo predominio de las rehabilitaciones en el maxilar inferior $(66,66 \%)$ con respecto al maxilar superior $(26,66 \%)$.

La distribución, en función del tipo de rehabilitación planificada, fue la siguiente:

Maxilar inferior:

Sobredentaduras: $23,07 \%$

Prótesis fija: $76,92 \%$

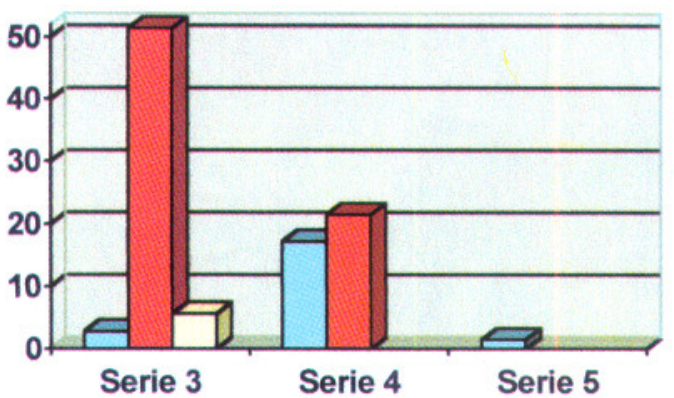

Fig. 12: Distribución de porcentajes según longitud y diámetro de los implantes utilizados.

Maxilar superior:

Sobredentaduras: $16,66 \%$

Prótesis fija: $83,33 \%$

\section{Cantidad y calidad óseas:}

La determinación de ambas variables mediante registros radiográficos, establecieron los siguientes porcentajes:

Cantidad: A (58,33\%), B (8,33\%), C (25\%), D (8,33\%), E ( 0\%)

Calidad: Tipo 1 (0\%), Tipo 2 (46,15\%), Tipo 3 (46,15\%), Tipo $4(7,69 \%)$.

\section{Diámetro y longitud de los implantes (Fig. $\left.n^{\circ} 12\right)$ :}

Los implantes Defcon ${ }^{\circledR}$ utilizados correspondieron a la serie 3 (diámetro $3,4 \mathrm{~mm}$ ), serie 4 (diámetro $4 \mathrm{~mm}$ ) y serie 5 (diámetro $5,3 \mathrm{~mm}$ ).

La distribución por porcentajes fue de:

Serie 3: $60 \%$.

Serie $4: 38,57 \%$.

Serie 5: $1,42 \%$.

Las longitudes pertenecientes a cada una de las series fueron de:

\section{Serie 3:}

Implantes de $10 \mathrm{~mm}$ : $2 \quad(2,85 \%)$.

Implantes de $13 \mathrm{~mm}: 36 \quad(51,42 \%)$

Implantes de $15 \mathrm{~mm}: \quad 4 \quad(5,71 \%)$

Serie 4:

Implantes de $10 \mathrm{~mm}: 12(17,14 \%)$.

Implantes de $13 \mathrm{~mm}: 15(21,42 \%)$.

Serie 5:

Implantes de $10 \mathrm{~mm}: 1$ (1,42\%). 


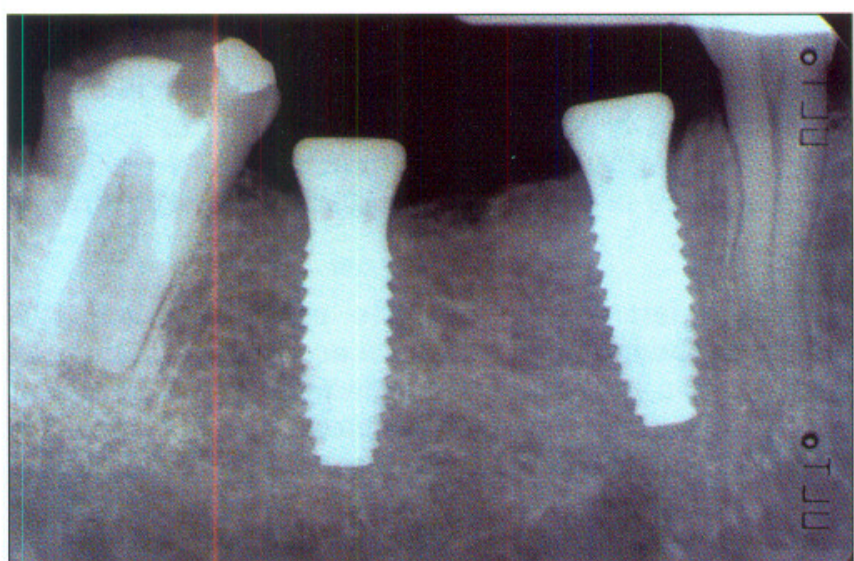

Fig. 13: Control radiográfico (Rx Periapical) en el que se observa la correcta osteointegración con ligera pérdida ósea alrededor de la cabeza.

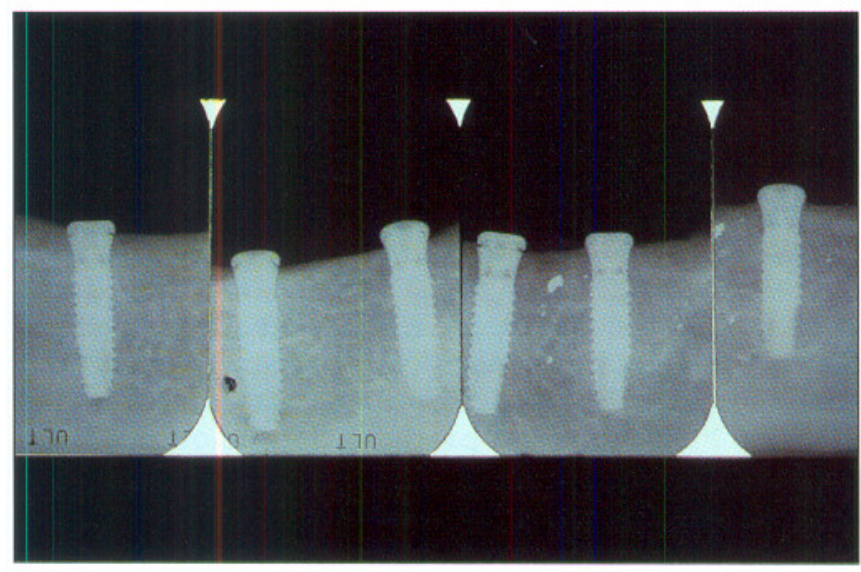

Fig. 14: Control radiográfico (Rx. Periapical) sobre paciente con cuatro implantes para rehabilitación mediante sobredentadura.

\section{Control postoperatorio y evolución:}

Una vez intervenidos, se prescribió el tratamiento habitual (antibioterapia, analgésicos-antiinflamatorios), y se solicitó a todos los integrantes del estudio que realizasen una primera revisión a las cuarenta y ocho horas, observándose síntomas habituales como inflamación (60\%) y hematomas $(33,33 \%)$.

Posteriormente y coincidiendo con la retirada de la sutura (diez días) se comprobó que la inflamación había desparecido en la totalidad de los casos existentes y la evolución de los hematomas seguía el curso habitual.

Se dieron las instrucciones de higiene y mantenimiento, transmitiendo al paciente la necesidad de realizar revisiones posteriores al mes y mes y medio, antes de proceder al estudio radiológico.

Durante este tiempo no se detectaron complicaciones

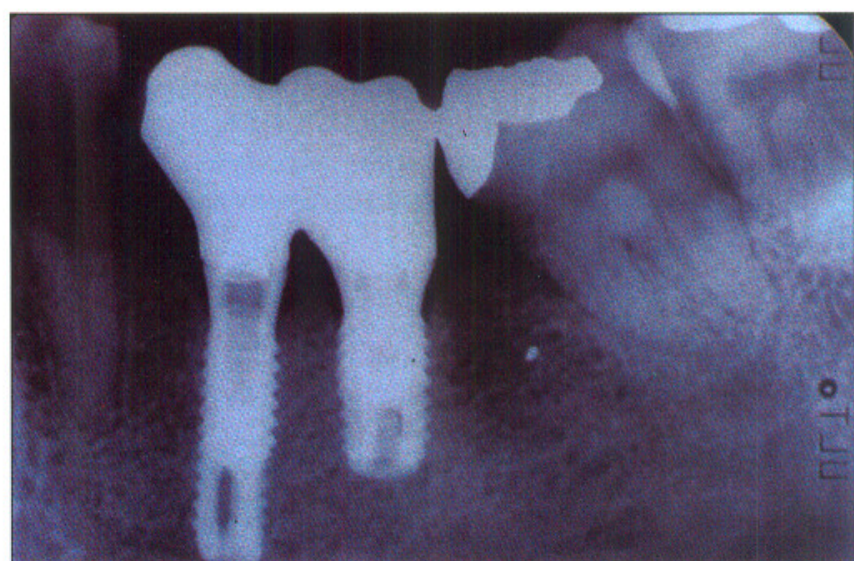

Fig. 15: Control radiográfico (Rx. Periapical) a los seis meses de carga observándose un comportamiento adecuado.

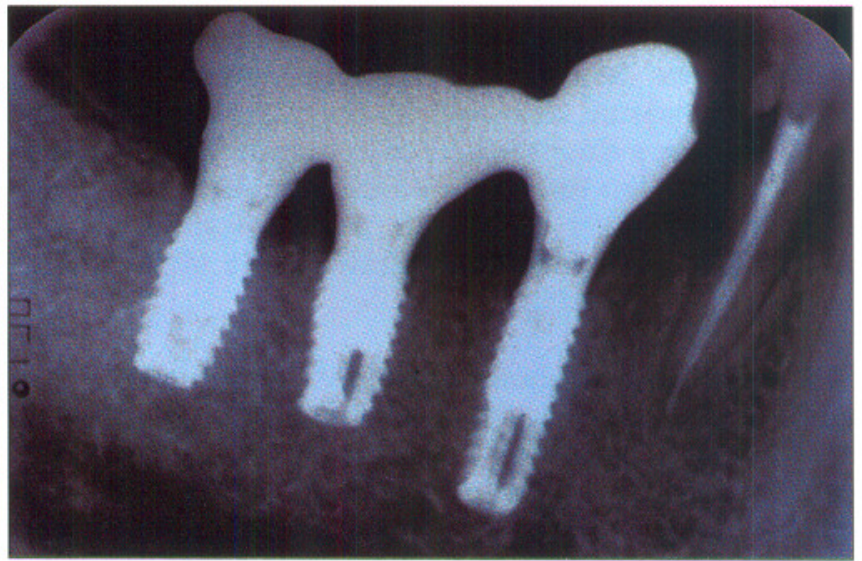

Fig. 16: Control radiográfico (Rx. Periapical) a los seis meses de carga en extremo libre inferior.

y tan solo se observó en el $21,42 \%$ de los casos la exposición parcial de los tornillos de cierre que correspondieron a cuatro implantes de la serie 3, 10 implantes de la serie 4 y un implante de la serie 5.

\section{Resultados radiológicos y valoración del sistema:}

A todos los pacientes, incluidos aquellos que presentaban exposición de los tornillos de cierre, se les realizó estudios radiológicos, mediante técnica panorámica y proyecciones periapicales, junto a exploración periodontal durante la colocación de las cabezas de cicatrización.

Los resultados evidenciaron tres casos de fracaso $(4,28 \%)$, pertenecientes a dos implantes de la serie $4 \mathrm{y}$ al único de la serie 5 que se utilizó. Cada uno de los implantes perdidos pertenecía a pacientes distintos sobre los cuales se habían colocados otros implantes que si se encontraban correctamente osteointegrados. 
Con respecto a los sesenta y siete implantes $(95,71 \%)$ que cumplían los criterios de éxito encontramos que en un $61,42 \%$ había una pérdida de dos milímetros sobre la cabeza del implante mientras que en el $39,57 \%$ restante el recubrimiento óseo fue total. (Figs. $\left.\mathrm{n}^{\circ} 13-16\right)$

\section{DISCUSIÓN}

Los implantes de carga precoz representan, en la actualidad, una alternativa importante gracias a los modernos tratamientos de las superficies implantarias.

Los recubrimientos con hidroxiapatita, plasma de titanio, etc., han demostrado buenos resultados como tratamiento de adhesión a los implantes mejorando sustancialmente la interfase implante-tejido óseo. Sin embargo, existen desventajas como las de favorecer la liberación y desprendimiento de partículas de titanio durante las fuerzas de cizallamiento y tracción -caso del plasma de titanio- o de microfracturas y mayor facilidad en la contaminación de la superficie -caso de la hidroxiapatita-.

Las superficies implantarias, tratadas mediante técnicas de sustracción como es el grabado ácido, tienden a aumentar la superficie de contacto entre el hueso y el implante reduciendo el tiempo de cicatrización ósea. La rugosidad que se produce en la superficie del implante se consigue realizando la inmersión de los implantes en disoluciones ácidas que acaban atacando la superficie del titanio y provocando una rugosidad de aproximadamente dos micrómetros.

Las células osteogénicas proliferan y se diferencian en mejores condiciones sobre superficies rugosas que sobre lisas a la vez que su migración se ve favorecida gracias a una mayor estabilidad del coágulo y consecuentemente de la red de fibrina, resultados avalados por los estudios realizados, entre otros, por Wennerberg, Baker y Lazzara ${ }^{5-7}$.

El estudio preliminar que presentamos sobre nuestra experiencia en una nueva superficie implantaria, añade al grabado ácido la técnica de pasivado químico cuyo objetivo es aumentar la resistencia a la corrosión del implante. La inmersión en disoluciones ácidas con ligero carácter oxidante crea una capa inerte y estable de óxido de titanio de aproximadamente 5 a 10 micrómetros. Las ventajas que se consiguen con respecto a otras superficies son fundamentalmente la de mejorar la corrosión electroquímica en más de diez veces respecto a las muestras sin pasivar. También se reduce a la mitad la liberación de iones de titanio al medio, así como un aumento en la dureza superficial en más de 300 unidades Vickers, lo que produce una mayor resistencia al rayado y a la deformación durante su utilización clínica. Por último, esta superficie, presenta una capa homogénea y uniforme que no altera la rugosidad producida por el grabado ácido.

El comportamiento clínico de estos implantes ha resultado satisfactorio, teniendo en cuenta que en esta primera fase los resultados obtenidos han sido de un $95,71 \%$ de éxito, lo que les sitúa en proporciones similares a otros sistemas de implantes.

Cabe destacar que, bajo nuestra opinión, se trata de un sistema sencillo, cómodo y práctico a la hora de realizar el lecho óseo para la colocación del implante, a la vez que cumple con las necesidades requeridas para cualquier situación antómica, dada las diferentes disponibilidades de implantes en cuanto a diámetros y longitudes.

El análisis de los fracasos obtenidos (3 casos $4,28 \%)$, nos conduce una vez más a reflexiones que, en ocasiones, no pueden ser contestadas bajo una lógica evidente. De un lado, la longitud y el diámetro de los implantes, dos de la serie 413 y uno de la serie 510, parece un hecho a tener en cuenta pues el diseño del lecho óseo y su posterior inserción -mayores longitudes y diámetros-, en determinados tipos de hueso pueden producir condiciones adversas para la osteointegración. También es importante resaltar, sobre este mismo punto que la cantidad y calidad óseas presentadas por los pacientes eran variadas, siendo en algunos casos no demasiado favorables como lo demuestran los porcentajes -cantidad D $(8,33 \%)$ - calidad $3(46,15 \%)$ - calidad 4 $(7,69 \%)$-. Finalmente, también nos planteamos el interrogante del porqué ha existido este fracaso sobre tres implantes en tres pacientes diferentes en los que idénticas superficies implantarias, en los mismos pacientes, han respondido favorablemente en la región maxilar o mandibular y en donde tan solo se han diferenciando en siete milímetros de distancia de su inserción.

La evolución postoperatoria, ha resultado compatible con los estándares habituales y tan solo reflejar el comportamiento mucoso ante los sistemas de implantes que se caracterizan por su conexión con hexágono externo que hace que muchas veces se produzcan descubrimientos de los tornillos de cierre lo que para algunos profesionales puede no ser de su agrado, o incluso crear sensación de alarma para los pacientes. En estas situaciones, cabe expresar aún más la necesidad de higiene de los pacientes, no debiendo ser ningún factor negativo -si se cumplen entre otros estos requisitos- para una correcta osteointegración. 
El hecho de que todos los implantes fueran cargados a las dos meses ha constituido una gran ventaja para los pacientes. El diseño protético basado en el sistema multiconexión de este sistema hace que el comportamiento de las prótesis pueda resultar altamente satisfactorio. Sin embargo, el carácter preliminar de nuestro estudio no nos permite todavía aseverar estas afirmaciones, por lo que creemos que estudios más amplios y a mayor tiempo deberán o no refrendar nuestros resultados.

\section{SUMMARY}

We are submitting a study on a new implant system in which the surface of the implant is treated by means of a process of acid etching and chemical passivation. The fifteen patients that were included in this assay were given a total of seventy implants according to the different states and conditions of bone quality and quantity. After the first stage the results showed a success rate of $95.71 \%$, which means that prothesis loading can be effected within a period of approximately two months, and which has led us to stablish, by way of preliminary conclusions, that this type of implant surface means that the waiting time for our patients can be reduced, and that results similar to those obtained using other, more conventional surfaces can be achieved.

\section{KEY WORLDS}

Implants. Acid etching. Chemical passivation. Early loading.

\section{BIBLIOGRAFÍA}

1. Ledermann PD. Stegprothetische Versorgung des zahnlosen Unterkiefers mit Hilfe plasmabeschichteten Titanschraubimplantaten. Deutsche Zahnartzlische Zeitung. 1979; 34: 907-11.

2. Babbusch CA, Kent J, Misiek D. Titanium plasma-sprayed (TPS) screw implants for the reconstruction of the edentulous mandible. J Oral Maxilofac Surg. 1986; 44: 274-82.

3. Buser D, Schenk RK, Steinemann SG, Fiorellini JP, Fox $\mathrm{CH}$, Stich H. Influence of surface characteristics on bone integration of titanium implants: A histomorphometric study in miniature pigs. J Biomed Mat Res. 1991; 25: 889902.

4. Chiapasco M, Gatti C, Rossi E, Haefliger W, Markwalder TH. Implant-retained mandibular overdentures with inmediate loading. A retrospective multicenter study on 226 consecutive cases. 1997; 8: 48-57.

5. Wenneberg $\AA$, Hallgren $C$, Johansson C, Danielli S. A histomorphometric evaluation of screw-Shaped implants each prepared with two surface rougnesses. Clin Oral Implant Res. 1998; 9: 11-9.

6. Lazzara R, Porter S, Testori T, Galante J, Zetterqvist L. A prospective multicerter study evaluating loading of osseotite implants two months after placement: one year results. J Est Dent. 1998; 10 (6): 280-9.

7. Baker DA, London $\mathrm{RH}, \mathrm{O}^{\prime} \mathrm{Neal}$ RB. Integration strength and speed of dual-etched titanium implants: a comparative study in rabbits. Int J Oral Maxillofac Impl. 1999; 14 (5): 722-7. 